\title{
Unphosphorylated STAT3 in heterochromatin formation and tumor suppression in lung cancer
}

Pranabananda Dutta ${ }^{1 \dagger}$, Lin Zhang ${ }^{1 \dagger}$, Huijun Zhang ${ }^{1,2}$, Qin Peng ${ }^{3}$, Phillippe R. Montgrain ${ }^{1,4}$, Yingxiao Wang ${ }^{3}$, Yuanlin Song ${ }^{2}$, Jinghong $\mathrm{Li}^{\mathrm{i}^{*}}$ and Willis X. $\mathrm{Li}^{i^{*}}$ (D)

\begin{abstract}
Background: Aberrant JAK/STAT activation has been detected in many types of human cancers. The role of JAK STAT activation in cancer has been mostly attributed to direct transcriptional regulation of target genes by phosphorylated STAT (pSTAT), while the unphosphorylated STAT (USTAT) is believed to be dormant and reside in the cytoplasm. However, several studies have shown that uSTATs can be found in the nucleus. In addition, it has been shown that tissue-specific loss of STAT3 or STAT5 in mice promotes cancer growth in certain tissues, and thus these STAT proteins can act as tumor suppressors. However, no unifying mechanism has been shown for the tumor suppressor function of STATs to date. We have previously demonstrated a non-canonical mode of JAK/STAT signaling for Drosophila STAT and human STAT5A, where a fraction of USTAT is in the nucleus and associated with Heterochromatin Protein 1 (HP1); STAT activation (by phosphorylation) causes its dispersal, leading to HP1 delocalization and heterochromatin loss.

Methods: We used a combination of imaging, cell biological assays, and mouse xenografts to investigate the role of STAT3 in lung cancer development.

Results: We found that USTAT3 has a function in promoting heterochromatin formation in lung cancer cells, suppressing cell proliferation in vitro, and suppressing tumor growth in mouse xenografts.

Conclusions: ThuS, USTAT3 possesses noncanonical function in promoting heterochromatin formation, and the tumor suppressor function of STAT3 is likely attributable to the heterochromatin-promoting activity of USTAT3 in the non-canonical JAK/STAT pathway.
\end{abstract}

Keywords: USTAT3, Heterochromatin, JAK/STAT, Lung cancer, FRET

\section{Background}

In the canonical JAK/STAT signaling pathway, activated JAK phosphorylates STAT at a tyrosine residue around a.a.700, and the resulting phosphorylated STAT (pSTAT) dimerizes and translocates to the nucleus, where it functions as a transcription factor, while the dormant unphosphorylated STAT (USTAT) in the cytoplasm has no significant functions. However, our previously research using Drosophila STAT92E and human

\footnotetext{
* Correspondence: jil055@health.ucsd.edu; wxli@health.ucsd.edu

†Pranabananda Dutta and Lin Zhang contributed equally to this work.

'Department of Medicine, University of California San Diego, La Jolla, CA 92093, USA

Full list of author information is available at the end of the article
}

STAT5A has demonstrated a non-canonical JAK/STAT signaling, in which USTAT is capable of associating with HP1 and stabilizing heterochromatin [1, 2]. JAK activation can increase pSTAT and decrease uSTAT, thus causing heterochromatin instability $[3,4]$. Other groups have shown that human JAK2 activation reduces heterochromatin in leukemia and stem cells [5-8].

Many groups have reported that uSTATs can translocate into and prominently exist in the nucleus in various mammalian cells at quiescence, when STAT proteins are not phosphorylated [9-16]. Specifically, it has been shown that STAT3 maintains a prominent nuclear presence independent of its tyrosine phosphorylation status in several mammalian cell lines $[12,13,16]$, and that

(c) The Author(s). 2020 Open Access This article is distributed under the terms of the Creative Commons Attribution 4.0 International License (http://creativecommons.org/licenses/by/4.0/), which permits unrestricted use, distribution, and reproduction in any medium, provided you give appropriate credit to the original author(s) and the source, provide a link to the Creative Commons license, and indicate if changes were made. The Creative Commons Public Domain Dedication waiver (http://creativecommons.org/publicdomain/zero/1.0/) applies to the data made available in this article, unless otherwise stated. 
uSTAT5 similarly is detected in the nucleus of serumstarved unstimulated cells, where STATs are not phosphorylated [11, 17, 18]. Further, USTAT1, 3, and 5 can bind to DNA [18-21] and to regulate gene transcription $[9,13,14,18]$.

Our previous work has shown that the STAT-HP1 interaction is mechanistically and functionally conserved in human cells for STAT5A [2], which is most homologous to Drosophila STAT92E [22, 23]. We have shown that both endogenous STAT5A and transfected uSTAT5A (or STAT5A ${ }^{\mathrm{Y} 694 \mathrm{~F}}$ ) are prominently present in the nucleus of cultured human cells [2]. This observation is consistent with reports by other groups (see Fig. 1a in ref. [11]; Fig. 5A in [17]; Fig. 1 in ref. [18]). In addition, uSTAT5A physically interacts with $\mathrm{HP} 1 \alpha$ via an HP1-binding motif, PxVxI, present in STAT proteins $[1,2]$. It has been shown that uSTAT5 in the nucleus directly binds to and represses differentiation genes in hematopoietic progenitor cells [18]. Thus, the "textbook" version of JAK/STAT signaling needs revision; uSTATs are not simply "latent cytoplasmic proteins" but constantly shuttle into the nucleus $[15,24]$, where they may function to regulate gene transcription $[14,18]$ and promote heterochromatin stability $[2,4,25]$.

While many groups have demonstrated nuclear localization of uSTATs, their nuclear functions are nonetheless less clear. Although it is reported that USTAT3 can activate gene expression [14], genomic studies have shown that uSTAT5 is mainly involved in gene repression, that activation of the JAK/STAT pathway causes genome-wide redistribution of chromatinbound STAT5 to traditional STAT transcriptional targets, due to conversion of uSTAT5 to pSTAT5, and that either STAT5 activation or its depletion causes derepression of differentiation genes [18]. This latter finding is consistent with our studies of STAT5A [2]. We have shown that USTAT5A functions strikingly similar to $\mathrm{HP} 1 \alpha$ in gene repression, and that many of the genes repressed by uSTAT5A and HP1 $\alpha$ in common are overexpressed in colon cancer [2]. Importantly, these same genes increase their expression when endogenous STAT5A or HP1 $\alpha$ is knocked down, suggesting that endogenous uSTAT5A and HP1 $\alpha$ are involved in repressing these genes possibly via heterochromatin formation.

Heterochromatin is important for chromosomal compaction and transcriptional silencing as well as for genome stability, animal longevity, and tumor suppression [26-28]. Cellular differentiation is associated with increases in heterochromatin levels [29-32]. The reverse of this process, i.e., dedifferentiation, is a hallmark of cancer development [33]. Loss of heterochromatin and derepression of satellite repeats is found in many cancers [34]. Tumorigenesis occurs only in cells that have decreased levels of heterochromatin or are unable to form new heterochromatin $[35,36]$. Heterochromatin is marked by di- or tri-methylated lys 9 on histone $\mathrm{H} 3$ (H3K9me3), which provides docking site for HP1, both of which are hallmarks of heterochromatin [26-28]. It has been shown that Suv39h1, a H3K9-specific histone methyl transferase (HMT) and key component of heterochromatin, functions as a tumor suppressor whose loss permits lymphoma development in response to oncogenic Ras [35]. Consistent with these findings, we have shown that heterochromatin is essential for maintaining genome stability [37], and suppresses tumor growth [2].

Since the HP1-binding motif PxVxI is conserved in all STAT proteins including STAT3, we sought to investigate whether uSTAT3 can also bind HP1 and can thereby play a role in tumor suppression. Activation of STAT3 has been found more often than STAT5 or any other STATs in cancers. Thus, understanding the functions of STAT3 is important in cancer biology. Interestingly, it has been shown that loss of STAT3 or STAT5 promotes cancer growth in certain tissues, suggesting these STAT proteins might function as tumor suppressors in these situations [38-42]. On the other hand, several groups have shown that increasing uSTAT3 levels inhibits tumor growth [43, 44], an effect that has been attributed to its dominantnegative interference with transcriptional activation of pSTAT3. The apparently contradicting results regarding STAT3 in lung cancer are entirely consistent with our hypothesis that the tumor suppressor function of STAT3 stems from its noncanonical function in controlling heterochromatin, i.e., uSTAT3 rather than pSTAT3 suppresses tumors. Our hypothesis predicts that expressing uSTAT3 and STAT3 knockdown would have opposite effects on lung cancer growth.

To understand the biological functions of STAT3 in lung cancer and to determine whether noncanonical function of STAT3 plays a role in lung cancer development, we investigated the interaction between STAT3 and HP1 $\alpha$ in a few non-small cell lung cancer (NSCLC) cell lines. We found that STAT3 and HP1 $\alpha$ partially colocalize in the nucleus and might physically interact, and that USTAT3 promotes heterochromatin formation and suppresses lung cancer cell proliferation in vitro and in vivo. These results suggest that the non-canonical functions of STAT3 operate in lung cancer cells, in which USTAT3 plays a role in suppressing cancer growth.

\section{Results}

Nuclear localization of USTAT3 and its physical interaction with HP1a

As has been previously reported, uSTAT3 is predominantly detected in the nucleus of various types of serumstarved, unstimulated cells (e.g., Fig. 1 in [12]). We 


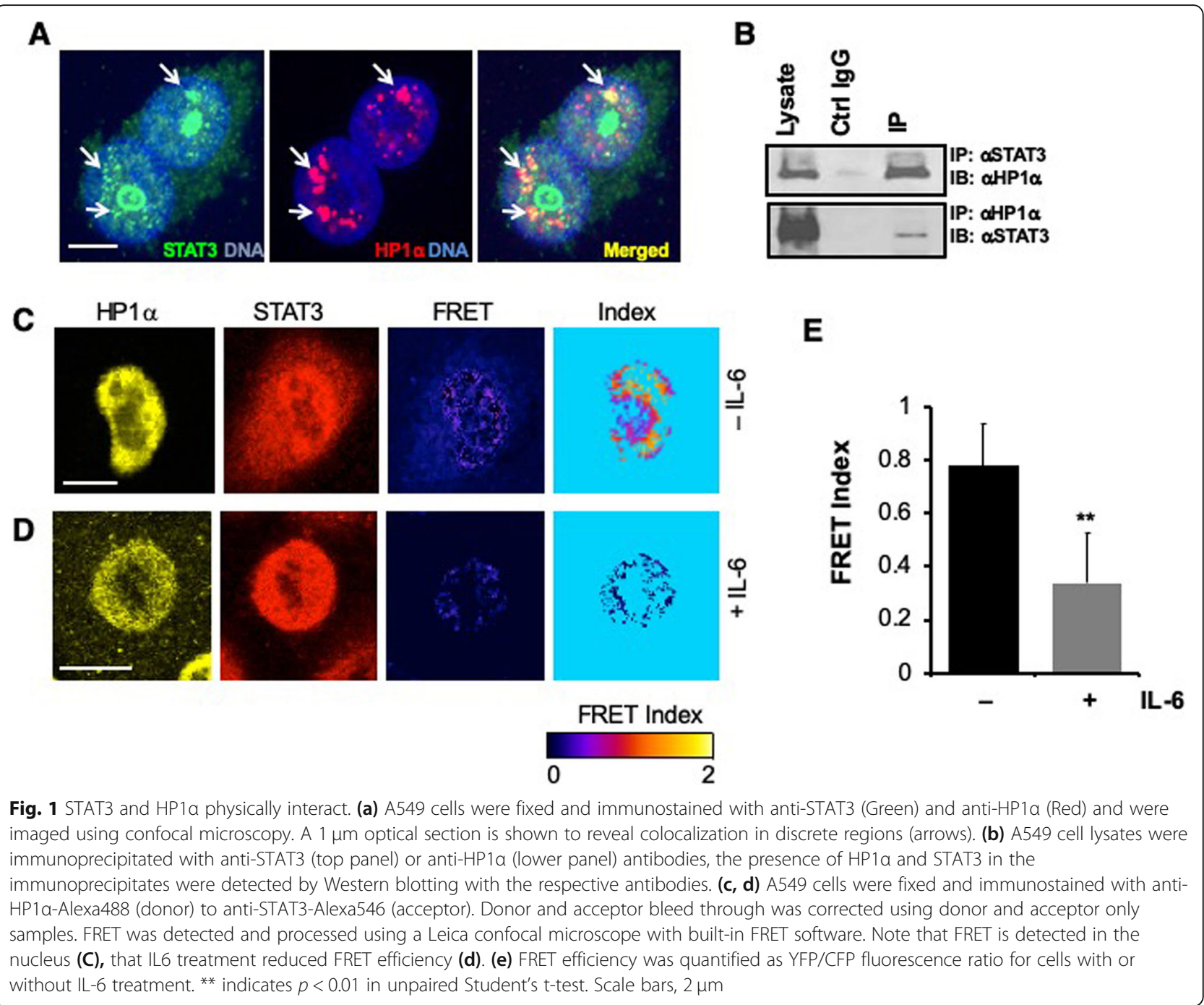

examined the subcellular localization of endogenous STAT3 and HP1 $\alpha$ in in serum starved cells using several lines of lung cancer cells including A549, H226, H441, H460, H520, as well as HeLa and HEK293T cells. When observed with a laser confocal microscope in thin optical sections, we found that endogenous STAT3 in all these cell lines can be detected in the nucleus as unevenly distributed particulates, some of which are colocalized with HP1 $\alpha$ (Fig. 1a). Some of these cancer cell lines show very low or no detectable pSTAT3 under unstimulated conditions, as previously reported (e.g., A549) [44]. The pattern of nuclear STAT3 distribution in these cells does not correlate with its phosphorylation status, consistent with previous reports that STAT3 is prominently detected in the nucleus regardless of its phosphorylation status $[12,13,16]$. In addition, we found that endogenous STAT3 and HP1 $\alpha$ co-immunoprecipitate (Fig. 1b), consistent with the idea that they may physically interact.
To investigate if the partially colocalized STAT3 and HP $1 \alpha$ in the nucleus indeed physically interact, we employed fluorescence resonance energy transfer (FRET), which is based on transfer of energy from one fluorescent molecule (donor) to another (acceptor) only when the two are in close proximity $(<10 \mathrm{~nm}$ apart), which occurs when the two molecules directly interact $[45,46]$. In order to investigate the proximity of unmodified endogenous STAT3 and HP1 $\alpha$ in cells, we used fluorophore-conjugated secondary antibodies and FRET $[46,47]$ in fixed cells. We found high FRET efficiency from anti-HP1 $\alpha$-Alexa488 (donor) to antiSTAT3-Alexa546 (acceptor) only in discrete regions of the nucleus (Fig. 1c), suggesting that STAT3 and HP1 $\alpha$ physically interact in these regions of the nucleus. Importantly, when treated with the cytokine IL6, which activates STAT3 by phosphorylation, increasing pSTAT3 and decreasing uSTAT3, we found that FRET efficiency was significantly reduced, suggesting a loss of STAT3- 
HP1 $\alpha$ interaction (Fig. 1d, e). The FRET results suggest that the colocalized USTAT3 and HP1 $\alpha$ physically interacts.

\section{USTAT3 promotes heterochromatin formation}

To investigate if by binding to HP1 $\alpha$, uSTAT3 is able to promote heterochromatin formation, we carried out the following experiments. First, using Western blotting, we found that expressing an unphosphorylatable mutant STAT3 (STAT3 ${ }^{\mathrm{Y} 705 \mathrm{~F}}$ ), representing uSTAT3, causes a notable increase in the levels of the heterochromatin mark, H3K9me3, whereas depleting endogenous STAT3 leads to moderately reduced H3K9me3 levels (Fig. 2a). Second, by using immunostaining, we found that expressed STAT3 $^{\mathrm{Y} 705 \mathrm{~F}}$ increased H3K9me3 levels, whereas expressing the non-HP1-interacting STAT3 $^{\mathrm{V} 462 \mathrm{~A}}$ had no effects (Fig. 2b). Third, we employed a FRET-based heterochromatin sensor to investigate the effects of HP1 $\alpha$ and USTAT3 on global heterochromatin levels. The heterochromatin FRET sensor consists of peptides of $\mathrm{H} 3$ and HP1 fused to YFP and CFP, respectively, which has been previously used as a robust readout of H3K9me3 levels when expressed by transfection [48-50]. We found that, as expected, altering $\mathrm{HP} 1 \alpha$ levels, by overexpressing $\mathrm{HP} 1 \alpha$ or expressing a small hairpin (sh) HP1 $\alpha$ RNA, affect global heterochromatin levels, as indicated by the FRET sensor (Fig. 2c, d). Importantly, we found that expressing STAT3 $3705 \mathrm{~F}$ increased, whereas knocking down STAT3 decreased global heterochromatin levels in A549 cells (Fig. 2c, d). A hallmark of non-canonical STAT function is that expressing unphosphorylatable mutant STAT has the opposite effect to knocking down STAT, whereas in the canonical pathway, they have the same effect. These
A

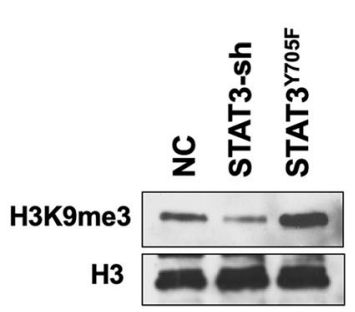

C

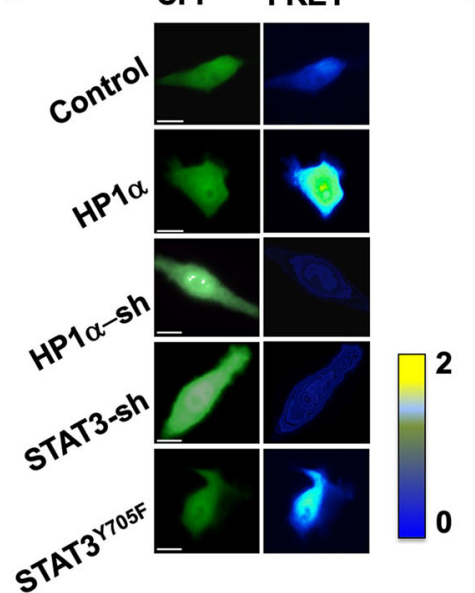

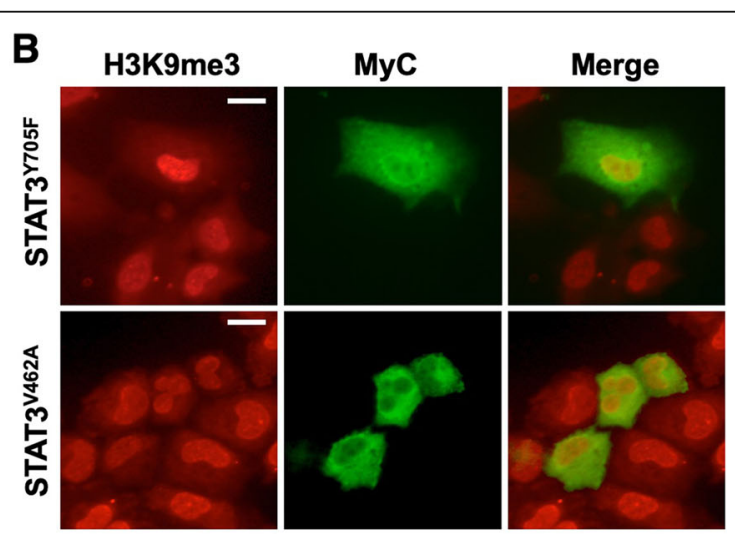

D

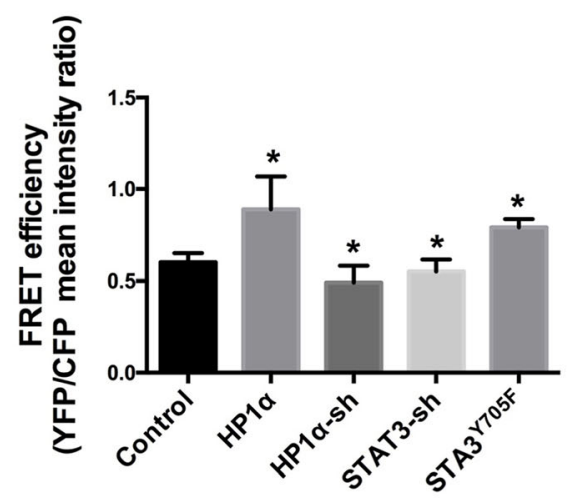

Fig. 2 uSTAT3 promotes heterochromatin formation in lung cancer cells. (a) Total lysates of A549 cells stably transfected with the indicated DNA constructs were subjected to SDS-PAGE and blotted with anti-H3K9me3. Note that expressing STAT3 ${ }^{\text {Y705F }}$ or shSTAT3 dramatically increased or moderately decreased H3K9me3 levels, respectively. sh: small hairpin RNAi; NC, non-targeting control. (b) A549 cells were transiently transfected with MyC-tagged STAT3 ${ }^{\text {Y705F }}$ (upper) or STAT3 ${ }^{\text {V462A }}$ (lower) were immunostained with anti-H3K9me3 (left) or Myc (middle). Note that STAT3 ${ }^{\text {Y705F }}$, but not STAT3 ${ }^{\mathrm{V} 462 \mathrm{~A}}$, expression increased H3K9me3, when compared with untransfected neighboring cells. (c,d) A549 cells stably expressing the indicated STAT3 or HP1a transgenes were transfected with a heterochromatin FRET sensor, consisting of an HP1-H3 peptide with CFP and YFP. (c) Representative FRET images of cells with indicated transgene expression. (d) Mean FRET efficiency (YFP/CFP fluorescence ratio) with standard deviations was calculated for each group. * indicates $p<0.05$ in Student's t-test. Scale bars, $2 \mu \mathrm{m}$ 
results suggest that STAT3 also has non-canonical function in regulating heterochromatin globally.

To evaluate the changes in cellular phenotypes from altering heterochromatin, we carried out the following two experiments. First, we investigated cellular senescence, a state of irreversible cell cycle arrest associated with formation of specialized facultative heterochromatin, which is hypothesized to repress proliferationpromoting genes and suppress tumor development [35, 51]. Senescent cells are commonly detected by histochemical staining for senescence-associated $\beta$ galactosidase (SA- $\beta$ gal) [52]. Using stable A549 cells, we found that expressing uSTAT3 or HP1 $\alpha$ led to more senescent cells, whereas knocking STAT3 or HP1 $\alpha$ resulted in fewer senescent cells (Fig. 3a, b). Second, heterochromatin is known to repress transcription from satellite repeats, whose derepression is found in many cancers [34]. Using quantitative real-time polymerase chain reaction (qRT-PCR), we found that USTAT3 and HP1 $\alpha$ repressed major satellite transcripts, whereas knocking down endogenous STAT3 or HP1 $\alpha$ caused a 4-fold increase in major satellite transcripts (Fig. 3c).

\section{uSTAT3 and HP1a affects lung cancer cell growth in vitro and in vivo}

To investigate whether uSTAT3 and HP1 $\alpha$ play roles in cancer growth, we assessed the effects of overexpressing these genes on lung cancer cell growth using stably transfected A549 lung cancer cells. We first used a clonogenic assay to assess the ability of single lung cancer cells to grow into colonies [54]. We found that, compared with control, A549 cells stably expressing STAT3shRNA had more colonies, whereas cells expressing uSTAT3 $\left(\right.$ STAT3 $^{\mathrm{Y} 705 \mathrm{~F}}$ ) or HP1 $\alpha$ had fewer colonies (Fig.

A
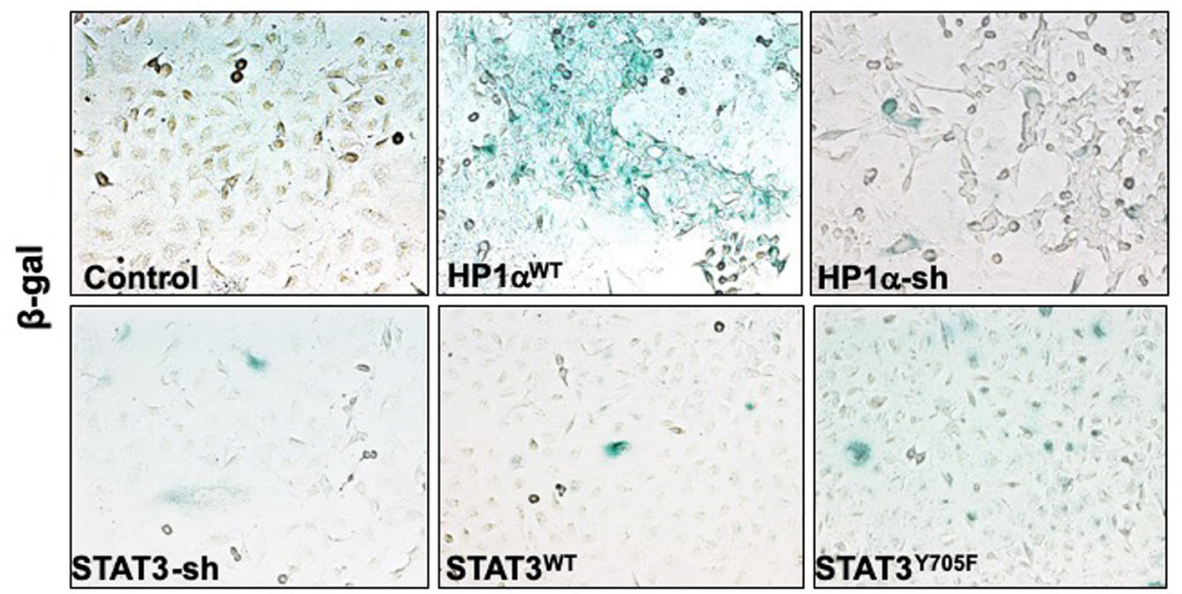

B

C
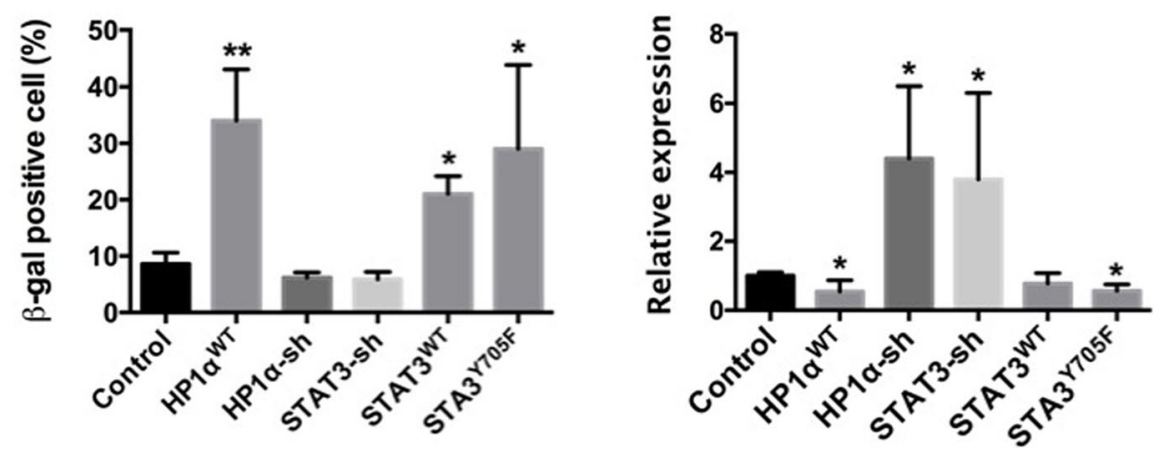

Fig. 3 STAT3 and HP1a affect cellular senescence and major satellite transcription. (a) Representative images are shown of A549 cells stably expressing the indicated STAT3 or HP1a transgenes assayed for senescence-associated beta-galactosidase (SA- $\beta$-gal; blue) at pH 6.0 [53]. Control cells were parent A549 cells without transgene expression. (b) Cell senescence was quantified by measuring senescence-associated $\beta$-Gal staining at an absorbance of $405 \mathrm{~nm}$. (c) Total RNA was isolated from A549 cells stably expressing the indicated transgene, and were quantified for major satellite transcripts using qRT-PCR. GAPDH gene transcript was used as the internal control for expression. The mean value \pm s.d. (standard deviation) was calculated in each group. ${ }^{*} P<0.05,{ }^{* *} P<0.01$ 
A

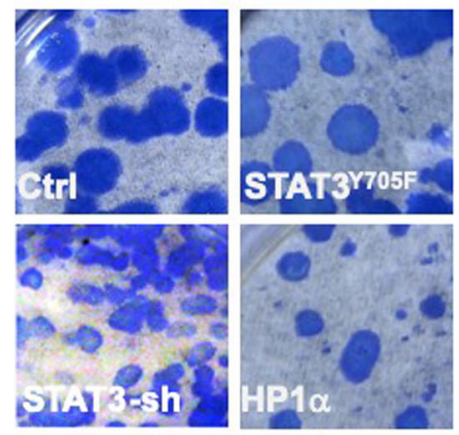

C
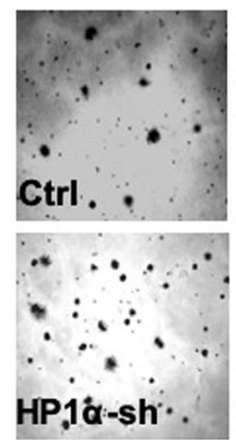

B

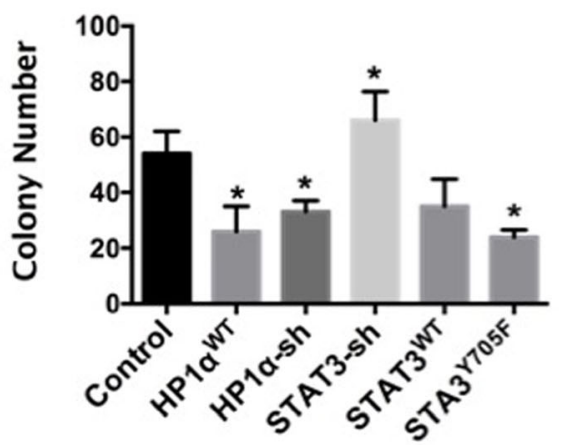

D

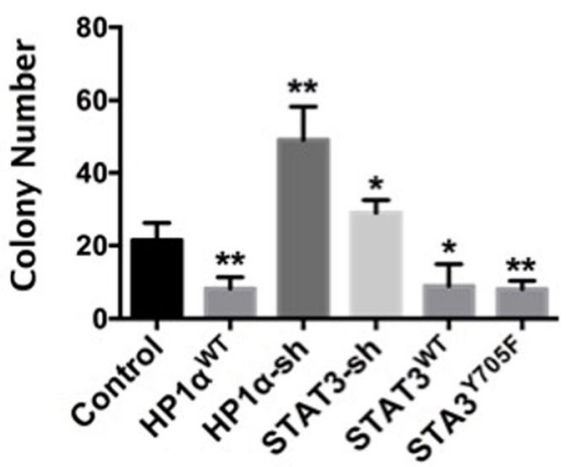

$\mathbf{E}$

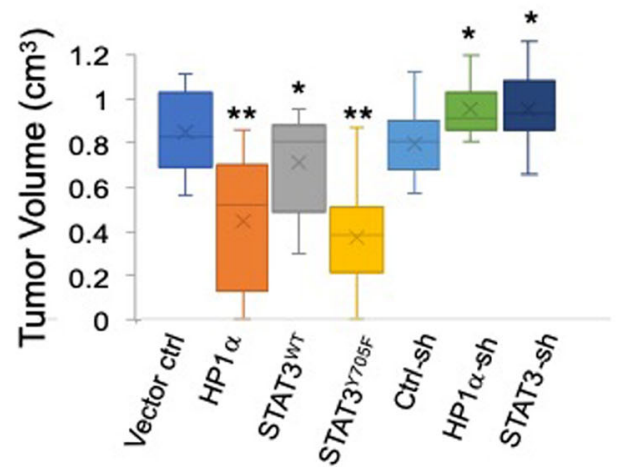

Fig. 4 STAT3 and HP1a affect lung cancer cell growth in vitro. A549 cells stably expressing the indicated STAT3 or HP1a transgenes were subjected to clonogenic assay $(\mathbf{a}, \mathbf{b})$ and soft-agar assay $(\mathbf{c}, \mathbf{d})$. Represented images are shown. Colony numbers are presented as the mean value \pm s.d. ${ }^{*}$ indicates $p<0.05$, ${ }^{*} P<0.01$, in Student's t-test. (e) Box plots of tumor volumes 4 weeks after subcutaneous injection of A549 cells stably expressing the indicated constructs are shown. Each box shows the range of the second and third quartiles of tumor volumes. The " $X$ " in the box indicates the median tumor volume. The bars ("whiskers") represent the largest and smallest tumors. Six injections $(n=6)$ were done for each of the indicated transgene and cell line combination. $n$ represents the number of injections for the indicated cell line. ${ }^{*}$ and ${ }^{*}$ indicate $p<0.05$ and $p<0.01$ (Student's t-test), respectively, when compared with the vector control or with control RNAi construct

$4 \mathrm{a}, \mathrm{b})$. We then used the soft-agar assay [55] to assess the effects of uSTAT3 and HP1 $\alpha$ on anchorageindependent growth of lung cancer cells. We found that knocking down STAT3 or HP1 $\alpha$ in A549 cells by expressing shRNAi constructs resulted in more and larger colonies, whereas overexpressing USTAT3 or HP1 $\alpha$ led to fewer and smaller colonies in soft agar (Fig. 4c, d). These results are consistent with the notion that
uSTAT3 and HP1 $\alpha$ suppress cancer cell growth by increasing heterochromatin levels. The discrepancy for HP1 $\alpha$-shRNA expression in the two different assays may involve differences in cell survival and will be further investigated.

Finally, we investigated the effects of uSTAT3 and HP1 $\alpha$ in cancer cell growth using mouse xenografts. We established lung cancer A549 cell lines stably expressing 
HP1 $\alpha$, STAT3 ${ }^{\text {WT }}$, STAT3 ${ }^{\text {Y705F }}$, and sh RNAi constructs targeting STAT3 and HP1 $\alpha$, respectively, as well as controls. We implanted these cells into immunodeficient (nude) mice subcutaneously and measured the growth of these cells as tumors in mice during the 4 weeks period after implantation. As shown at 4 weeks after implantation, we found that A549 cells expressing either HP1 $\alpha$ or STAT3 ${ }^{\mathrm{Y} 705 \mathrm{~F}}$ exhibited reduced capacity to grow as tumors, as indicated by the small tumor volumes at week four compared with A549 cells expressing vector control (Fig. 4e). Conversely, knocking down HP1 $\alpha$ or STAT3 by stably expressing RNAi constructs HP1 $\alpha$-sh or STAT3-sh resulted in larger tumor volumes (Fig. 4e). These results are consistent with a previous report that knocking down STAT3 in A549 cells promotes the growth of these lung cancer cells as mouse xenografts [38]. Taken together, our results suggest that uSTAT3 and $\mathrm{HP} 1 \alpha$ play roles in tumor suppression.

\section{Discussion}

We have previously discovered a noncanonical JAK/ STAT pathway, in which uSTAT plays a role in promoting heterochromatin formation by associating with HP1 $[1,3,4]$. We have further shown that the noncanonical function of STAT is conserved from Drosophila to human STAT5A [2]. The physiological functions of this noncanonical STAT pathway and how many other STAT proteins have this function, however, remain incompletely understood. In this study, we used human lung cancer cells to investigate the non-canonical functions of STAT3, which has been implicated in many human cancers and has been reported to also have tumor suppressor function. We have found that STAT3 possesses a noncanonical function, is capable of associating with HP1, promoting heterochromatin formation, and suppressing tumor progression.

We have shown by immunostaining and FRET that uSTAT3 and HP1 $\alpha$ colocalize, albeit partially, in the nucleus. The difference between uSTAT3 and uSTAT5A, which we have found to exhibit a more complete nuclear co-localization with $\mathrm{HP} 1 \alpha$ [2], is intriguing and is still under investigation. Nonetheless, our studies of STAT3, and previously of STAT5A, suggest that the noncanonical function of uSTAT, initially identified in Drosophila, is conserved in mammals. These results are consistent with our previous report that knocking down HP1 $\alpha$ enhances growth of tumor cells as mouse xenografts [2], and are consistent with reports that STAT3 functions as a tumor suppressor and loss of STAT3 promotes lung cancer cell growth in mouse xenografts [38]. Importantly, in this study we have shown that expressing uSTAT has effects opposite to knock downing STAT on cancer cell proliferation and tumor growth. Thus, our model that pSTAT promotes cancer development and
uSTAT suppresses tumors by influencing heterochromatin dynamics can reconcile the contradicting results reported by different groups regarding the functions of STAT proteins, especially STAT3, in cancers.

Furthermore, how heterochromatin loss might lead to cancer development and how heterochromatin is initially established remain unclear at the molecular level [27, 28]. Noncoding RNA transcription is essential for heterochromatin formation in fission yeast $[56,57]$. In other eukaryotes, however, DNA-binding proteins, including transcription factors, may initially bind to certain nucleation centers to recruit HMTs and/or HP1 [58-61]. Although BRCA1 and RB, and KLF11 can recruit HP1 to specific loci or maintain constitutive heterochromatin [58, 62-65], uSTAT recruiting HP1 to initiate new heterochromatin is yet to be investigated. It is conceivable that USTAT is among the factors that can initiate heterochromatin formation by binding to specific DNA sequence motifs and recruiting HP1. Although several transcription factors have been implicated in heterochromatin formation across diverse species [58, 62-65], a prominent heterochromatin localization has only been shown for STAT proteins [1,2]. Thus, STAT proteins might be among the protein factors that collectively or cooperatively control heterochromatin formation, which should have implications in both cancer biology and heterochromatin formation. The roles of uSTAT in heterochromatin initiation and maintenance and in cancer development are currently under investigation.

\section{Conclusions}

We have shown that STAT3 possesses the noncanonical function by which uSTAT3 promotes heterochromatin formation. We propose that the tumor suppressor function of STAT3 is likely attributable to the heterochromatin-promoting activity of uSTAT3 in the non-canonical JAK/STAT pathway.

\section{Methods \\ Source of cell lines}

Human NSCLC cell lines A549, H226, H441, H460, and H520; and other human cancer cell lines HeLa and HEK293T were from American Type Culture Collection (ATCC, Manassas, VA 20110 USA).

\section{Cell culture, DNA constructs, and transfection}

Human NSCLC cell lines A549, H226, H441, H460, and H520; and other human cancer cell lines HeLa and HEK293T cells were maintained in RPMI medium (Gibco) supplemented with $10 \%$ (v/v) FBS and antibiotics at $37^{\circ} \mathrm{C}$ and with $5 \% \mathrm{CO}_{2}$ in water-jacketed, humidified incubators. Transient transfection with plasmid DNA was done by using FuGENE 6 (Life Sciences, Inc) according to the manufacturer's protocol. Cells stably 
transfected with the indicated cDNAs or shRNAs were selected as puromycin $(5 \mu \mathrm{g} / \mathrm{ml})$-resistant colonies and several colonies were pooled. Cell culture and transfection procedure was approved by UCSD BUA R1347.

Plasmid DNA constructs with Myc-tagged human STAT3 and STAT3 ${ }^{\text {Y705F }}$ were kindly provided by Dr. Pradipta Ghosh (UCSD). DNA construct for sh-HP1 $\alpha$ was from Open BioSystems. The following DNA constructs were acquired from Addgene (Cambridge, MA): human HP1 $\alpha$ (17652), sh-STAT3 (26596), and H3K9me3 FRET reporter (22866).

\section{Immunostaining, Immunoprecipitation, and Western blotting}

For immunofluorescence, transfected cells were fixed in $4 \%$ paraformaldehyde for $10 \mathrm{~min}$, permeabilized in $0.2 \%$ Triton X-100 in phosphate-buffered saline (PBS) for 15 min, blocked with $5 \%$ bovine serum albumin (BSA) in PBS for $30 \mathrm{~min}$, and incubated overnight at $4{ }^{\circ} \mathrm{C}$ in primary antibodies. Primary antibodies used in this study include rabbit anti-STAT3 (1:250; Santa Cruz, sc-482), mouse anti-HP1 (CBX5) (1:200; Life Sciences, 730,019), goat anti-c-Myc (1:500, Fisher, NB600-335). Slides were washed four times in PBS and then immunostained with Alexa Fluor ${ }^{\ominus}$ 546-conjugated secondary antibody (1:250; Molecular Probes) at $37^{\circ} \mathrm{C}$ for $1 \mathrm{~h}$.

For co-immunoprecipitation, A549 cells were harvested in lysis buffer (50 mM Tris- $\mathrm{HCl}, \mathrm{pH} 8.0,250 \mathrm{mM}$ $\mathrm{NaCl}, 5 \mathrm{mM}$ EDTA, $1 \mathrm{mM}$ DTT, 5\% Glycerol) supplemented with complete protease inhibitor cocktail and PMSF at a final concentration of $2 \mathrm{mM}$. Portions of lysate containing equal amounts of protein $(200 \mu \mathrm{g})$ were then immunoprecipitated overnight at $4{ }^{\circ} \mathrm{C}$ with protein G-Agarose (Roche) bound antibodies. The beads were then washed six times with lysis buffer, and associated protein complexes were recovered in SDS sample buffer. Protein samples were resolved on a $10 \%$ sodium dodecyl sulphate-polyacrylamide gel and transferred onto Pure Nitrocellulose Blotting Membranes (Pall) for immunoblot analysis. Rabbit anti-STAT3 (1:250; Santa Cruz, sc482) and mouse anti-HP1 (CBX5) (1:200; Life Sciences, $730,019)$ primary antibodies were used to detect endogenous HP1 $\alpha$ and STAT3, respectively, followed by incubation with horseradish peroxidase (HRP) conjugated secondary antibodies and visualization using an enhanced chemiluminescence kit (Pierce). Full-length gel images are shown in Fig. S1.

\section{Fluorescence resonance energy transfer (FRET)}

To detect changes in histone methylation (H3K9me3) levels in living cells, cells were transfected with a CFP/ YFP histone methylation FRET reporter construct [48]. Donor and acceptor bleed through was corrected using donor and acceptor only samples. FRET measurements were carried out using a Zeiss Axio Observer fluorescence microscope equipped with FRET setup and software.

To detect FRET in fixed cells, cells were fixed on a coverslip and immunostained with anti-HP1 $\alpha$-Alexa488 (donor) to anti-STAT3-Alexa546 (acceptor) primary and secondary antibody pairs, as described in [47]. Donor and acceptor bleed through was corrected using donor and acceptor only samples. FRET was detected and processed using a Leica confocal microscope with builtin FRET software.

\section{Senescence-associated beta-galactosidase assay}

Cells were plated on 24 well plates. Nearly confluent cells were fixed with $3.7 \%$ Paraformaldehyde in PBS. $500 u$ of $\beta$-gal staining solution $(0.1 \% \mathrm{X}$-Gal, $5 \mathrm{mM} 5$ $\mathrm{mM}$ potassium ferrocyanide, $5 \mathrm{mM}$ potassium ferricyanide, $150 \mathrm{mM}$ Sodium chloride, and $2 \mathrm{mM}$ Magnesium chloride in $40 \mathrm{mM}$ citric acid/sodium phosphate solution, $\mathrm{pH}$ 6.0) was added and plates were incubated at $37^{\circ} \mathrm{C}$ overnight. After removal of staining solution, $70 \%$ glycerol was added and visualized with $20 \mathrm{X}$ bright field microscope. Senescence cells show blue staining in the cytosol [53].

\section{Quantitative PCR measurement of major satellite transcripts}

Total RNA from A549 cells stably expressing the indicated transgene was isolated using RNeasy Plus Mini kit (Qiagen) according to the manufacturer's manual. The SuperScript IV First-Strand Synthesis System (Thermo Fisher) was used to generate cDNA, according to the manufacturer's manual, and was subjected to Sybr Green qPCR using the Applied Biosystems 7300 Real Time PCR instrument per manufacturer's protocol. Major satellite transcripts expression values were normalized relative to Gapdh. Primers used for $\mathrm{qPCR}$ are as the following.

Major Sattellite Forward: AGGGAATGTCTTCCCATAAAAACT.

Major Satellite Reverse: GTCTACCTTTTATTTCAA TTCCCG.

Gapdh forward: CATGGGTGTGAACCATGAGA. Gapdh reverse: CAGTGATGGCATGGACTGTG.

\section{Anchorage-independent growth (soft agar) assay}

Stable A549 cell lines with the indicated transgene were maintained in RPMI medium supplemented with $10 \%$ fetal bovine serum. Cells grown to $60 \%$ confluency were resuspended in $0.4 \%$ Noble agar (Sigma, St. Louis, MO) in RPMI, and were seeded at a density of $1.5 \times 10^{5}$ cells/ well in 6-well culture plates on top of a $2 \mathrm{ml}$ underlayer composed of $0.8 \%$ agarose in RPMI. Media were refreshed twice per week for 3 weeks, and then were 
stained with p-iodonitrotetrazolium violet (Sigma, St. Louis, MO) and photographed on an inverted compound microscope with phase contrast optics.

\section{Colony formation assay}

Stable A549 cell lines with the indicated transgene were maintained in RPMI medium supplemented with $10 \%$ fetal bovine serum. Cells grown to $60 \%$ confluency were harvested and diluted in fresh media, and seeded at 100 cells/plate in 6-well plates. Cells were maintained in RPMI medium (Gibco) supplemented with $10 \%$ (v/v) FBS and antibiotics at $37^{\circ} \mathrm{C}$ and with $5 \% \mathrm{CO}_{2}$ in a water-jacketed, humidified incubator until large colonies (in excess of 50 cells) were visible in control plates. Medium was removed and cells were fixed with a solution of $6 \%$ glutaraldehyde and $0.5 \%$ crystal violet in PBS, and the number of colonies was counted.

\section{Xenograft assays}

Tumor formation was assayed by xeno-implantation of genetically perturbed cells. Prior to xeno-implantation, transfected cells were grown for $48 \mathrm{~h}$ under standard culture conditions without selective drugs. $5 \times 10^{5}$ cells were subcutaneously injected into the left and right flanks of 4-6 month old female CD-1 nude mice (Crl: CD-1-Foxn $1^{\mathrm{nu}}$, Charles River Laboratories). Tumor volumes were measured weekly using a caliper for 4 weeks. Following final tumor measurements, mice were euthanized by $\mathrm{CO} 2$ inhalation per IACUC protocol. Tumor volume was calculated using the average of 3 measurements of the tumor radius and the formula Volume $=(4 /$ 3) $\pi r^{3}$. The statistical significance of differences in tumor size was determined by Student's $t$-test. The vertebrate animal protocol has been approved by UCSD Institutional Animal Care and Use Committee (IACUC) (Protocol Number: S13282).

\section{Supplementary information}

Supplementary information accompanies this paper at https://doi.org/10. 1186/s12885-020-6649-2

Additional file 1 Supplementary Figure S1. Full-length gel images for Figure 1B, 2A.

\section{Abbreviations}

FRET: Fluorescence Resonance Energy Transfer; HP1: Heterochromatin Protein 1; JAK: Janus kinase; NSCLC: Non-small cell lung cancer; PSTAT: Phosphorylated STAT; QRT-PCR: Quantitative real-time polymerase chain reaction; SA- $\beta$ gal: Senescence-associated $\beta$-galactosidase; STAT: Signal Transducer and Activator of Transcription; USTAT: Unphosphorylated STAT

\section{Acknowledgements}

We thank Pradipta Ghosh (UCSD) for providing STAT3 plasmid DNA constructs, and the UCSD School of Medicine Microscopy Core for imaging techniques and equipment, which is supported by a NINDS P30 grant (NS047101).
Authors' contributions

P.D., W.L., J.L., P.M., Y.S., Y.W. designed experiments, wrote the main manuscript text, P.D., L.Z., H.Z., P.Q., performed experiments and prepared the figures. All authors have read and approved the manuscript.

\section{Funding}

This study was supported in part by grants from the National Institutes of Health (R01GM131044 to W.X.L) and a Research Award from the American Thoracic Society (to J.L.). The funders had no role in designing and executing the experiments.

Availability of data and materials

- All data generated or analysed during this study are included in this published article [and its supplementary information files].

\section{Ethics approval and consent to participate}

Mouse xenograft experiments were conducted in accordance with $\mathrm{NIH}$ guidelines for the care and use of laboratory animals, and the vertebrate animal protocol has been approved by UCSD Institutional Animal Care and Use Committee (IACUC) (Protocol Number: S13282).

\section{Consent for publication}

Not Applicable.

\section{Competing interests}

The authors declare no competing interests.

\section{Author details}

${ }^{1}$ Department of Medicine, University of California San Diego, La Jolla, CA 92093, USA. Department of Pulmonary Medicine, Shanghai Respiratory Research Institute, Zhongshan Hospital, Fudan University, Shanghai, China. ${ }^{3}$ Department of Bioengineering, Institute of Engineering in Medicine, University of California San Diego, La Jolla, CA 92093, USA. Veterans Affairs San Diego Healthcare System, San Diego, CA CA92037, USA.

Received: 25 July 2019 Accepted: 17 February 2020

Published online: 22 February 2020

\section{References}

1. Shi S, et al. Drosophila STAT is required for directly maintaining HP1 localization and heterochromatin stability. Nat Cell Biol. 2008;10(4):489-96.

2. Hu X, et al. Unphosphorylated STAT5A stabilizes heterochromatin and suppresses tumor growth. Proc Natl Acad Sci U S A. 2013;110(25):10213-8.

3. Shi $\mathrm{S}$, et al. JAK signaling globally counteracts heterochromatic gene silencing. Nat Genet. 2006:38(9):1071-6.

4. Li WX. Canonical and non-canonical JAK-STAT signaling. Trends Cell Biol. 2008:18(11):545-51.

5. Dawson MA, et al. JAK2 phosphorylates histone H3Y41 and excludes HP1alpha from chromatin. Nature. 2009:461(7265):819-22.

6. Griffiths DS, et al. LIF-independent JAK signalling to chromatin in embryonic stem cells uncovered from an adult stem cell disease. Nat Cell Biol. 2011; 13(1):13-21.

7. Rui $L$, et al. Cooperative epigenetic modulation by cancer amplicon genes. Cancer Cell. 2010;18(6):590-605.

8. Rui $L$, et al. Epigenetic gene regulation by Janus kinase 1 in diffuse large Bcell lymphoma. Proc Natl Acad Sci U S A. 2016;113(46):E7260-7.

9. Chatterjee-Kishore M, Wright KL, Ting JP, Stark GR. How Stat1 mediates constitutive gene expression: a complex of unphosphorylated Stat1 and IRF1 supports transcription of the LMP2 gene. EMBO J. 2000;19(15):4111-22.

10. Marg A, et al. Nucleocytoplasmic shuttling by nucleoporins Nup153 and Nup214 and CRM1-dependent nuclear export control the subcellular distribution of latent Stat1. J Cell Biol. 2004;165(6):823-33.

11. Iyer J, Reich NC. Constitutive nuclear import of latent and activated STAT5a by its coiled coil domain. FASEB J. 2008;22(2):391-400.

12. Liu L, McBride KM, Reich NC. STAT3 nuclear import is independent of tyrosine phosphorylation and mediated by importin-alpha3. Proc Natl Acad Sci U S A. 2005;102(23):8150-5.

13. Yue H, Li W, Desnoyer R, Karnik SS. Role of nuclear unphosphorylated STAT3 in angiotensin II type 1 receptor-induced cardiac hypertrophy. Cardiovasc Res. 2010;85(1):90-9. 
14. Yang J, Stark GR. Roles of unphosphorylated STATs in signaling. Cell Res. 2008;18(4):443-51.

15. Vinkemeier U. Getting the message across, STAT! Design principles of a molecular signaling circuit. J Cell Biol. 2004;167(2):197-201.

16. Cimica V, Chen HC, lyer JK, Reich NC. Dynamics of the STAT3 transcription factor: nuclear import dependent on ran and importin-beta1. PLoS One. 2011;6(5):e20188.

17. Shin HY, Reich NC. Dynamic trafficking of STAT5 depends on an unconventional nuclear localization signal. J Cell Sci. 2013;126(Pt 15):3333-43.

18. Park HJ, et al. Cytokine-induced megakaryocytic differentiation is regulated by genome-wide loss of a uSTAT transcriptional program. EMBO J. 2016; 35(6):580-94.

19. Neculai $D$, et al. Structure of the unphosphorylated STAT5a dimer. J Biol Chem. 2005;280(49):40782-7.

20. Timofeeva OA, et al. Mechanisms of unphosphorylated STAT3 transcription factor binding to DNA. J Biol Chem. 2012;287(17):14192-200.

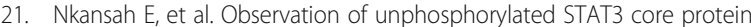
binding to target dsDNA by PEMSA and X-ray crystallography. FEBS Lett. 2013;587(7):833-9.

22. Hou XS, Melnick MB, Perrimon N. Marelle acts downstream of the Drosophila HOP/JAK kinase and encodes a protein similar to the mammalian STATs published erratum appears in cell 1996 Apr 19;85(2): following 290. Cell. 1996;84(3):411-9.

23. Yan R, Small S, Desplan C, Dearolf CR, Darnell JE Jr. Identification of a Stat gene that functions in Drosophila development. Cell. 1996;84(3):421-30.

24. Reich NC, Liu L. Tracking STAT nuclear traffic. Nat Rev Immunol. 2006;6(8): 602-12.

25. Xu N, Emelyanov AV, Fyodorov DV, Skoultchi Al. Drosophila linker histone H1 coordinates STAT-dependent organization of heterochromatin and suppresses tumorigenesis caused by hyperactive JAK-STAT signaling Epigenetics Chromatin. 2014;7:16.

26. Grewal SI, Elgin SC. Heterochromatin: new possibilities for the inheritance of structure. Curr Opin Genet Dev. 2002;12(2):178-87.

27. Grewal SI, Jia S. Heterochromatin revisited. Nat Rev Genet. 2007;8(1):35-46.

28. Wang J, Jia ST, Jia S. New insights into the regulation of heterochromatin. Trends Genet. 2016;32(5):284-94.

29. Panteleeva I, et al. HP1alpha guides neuronal fate by timing E2F-targeted genes silencing during terminal differentiation. EMBO J. 2007;26(15):3616-28.

30. Agarwal N, et al. MeCP2 interacts with HP1 and modulates its heterochromatin association during myogenic differentiation. Nucleic Acids Res. 2007;35(16):5402-8.

31. Grigoryev SA, Bulynko YA, Popova EY. The end adjusts the means: heterochromatin remodelling during terminal cell differentiation. Chromosom Res. 2006;14(1):53-69.

32. Olins DE, Olins AL. Granulocyte heterochromatin: defining the epigenome. BMC Cell Biol. 2005;6:39.

33. Sell S. Cellular origin of cancer: dedifferentiation or stem cell maturation arrest? Environ Health Perspect. 1993;101(Suppl 5):15-26.

34. Ting DT, et al. Aberrant overexpression of satellite repeats in pancreatic and other epithelial cancers. Science. 2011;331(6017):593-6.

35. Braig $\mathrm{M}$, et al. Oncogene-induced senescence as an initial barrier in lymphoma development. Nature. 2005;436(7051):660-5.

36. Narita $\mathrm{M}$, et al. A novel role for high-mobility group a proteins in cellular senescence and heterochromatin formation. Cell. 2006;126(3):503-14.

37. Yan SJ, Lim SJ, Shi S, Dutta P, Li WX. Unphosphorylated STAT and heterochromatin protect genome stability. FASEB J. 2011;25(1):232-41.

38. Grabner B, et al. Disruption of STAT3 signalling promotes KRAS-induced lung tumorigenesis. Nat Commun. 2015;6:6285.

39. Lee J, et al. Signal transducer and activator of transcription 3 (STAT3) protein suppresses adenoma-to-carcinoma transition in Apcmin/+ mice via regulation of Snail-1 (SNAI) protein stability. J Biol Chem. 2012;287(22): 18182-9.

40. Ferbeyre $\mathrm{G}$, Moriggl R. The role of Stat5 transcription factors as tumor suppressors or oncogenes. Biochim Biophys Acta. 2011;1815(1):104-14.

41. Yu JH, Zhu BM, Riedlinger G, Kang K, Hennighausen L. The liver-specific tumor suppressor STAT5 controls expression of the reactive oxygen speciesgenerating enzyme NOX4 and the proapoptotic proteins PUMA and BIM in mice. Hepatology. 2012;56(6):2375-86.

42. Zhang $Q$, Wang HY, Liu X, Wasik MA. STAT5A is epigenetically silenced by the tyrosine kinase NPM1-ALK and acts as a tumor suppressor by reciprocally inhibiting NPM1-ALK expression. Nat Med. 2007;13(11):1341-8.
43. Minami M, et al. STAT3 activation is a critical step in gp130-mediated terminal differentiation and growth arrest of a myeloid cell line. Proc Nat Acad Sci U S A. 1996;93(9):3963-6.

44. Song L, Rawal B, Nemeth JA, Haura EB. JAK1 activates STAT3 activity in nonsmall-cell lung cancer cells and IL-6 neutralizing antibodies can suppress JAK1-STAT3 signaling. Mol Cancer Ther. 2011;10(3):481-94.

45. Truong K, Ikura M. The use of FRET imaging microscopy to detect proteinprotein interactions and protein conformational changes in vivo. Curr Opin Struct Biol. 2001;11(5):573-8.

46. Snapp EL \& Hegde RS (2006) Rational design and evaluation of FRET experiments to measure protein proximities in cells. Curr Protoc Cell Biol chapter 17:unit 17 19.https://doi.org/10.1002/0471143030.cb1709s32

47. Waterhouse BR, et al. Assessment of EGFR/HER2 dimerization by FRET-FLIM utilizing Alexa-conjugated secondary antibodies in relation to targeted therapies in cancers. Oncotarget. 2011:2(9):728-36.

48. Lin CW, Jao CY, Ting AY. Genetically encoded fluorescent reporters of histone methylation in living cells. J Am Chem Soc. 2004;126(19):5982-3.

49. Tan Y, et al. Matrix softness regulates plasticity of tumour-repopulating cells via H3K9 demethylation and Sox2 expression. Nat Commun. 2014;5:4619.

50. Peng Q, et al. Coordinated histone modifications and chromatin reorganization in a single cell revealed by FRET biosensors. Proc Natl Acad Sci U S A. 2018:115(50):E11681-90.

51. Campisi J. Aging, cellular senescence, and cancer. Annu Rev Physiol. 2013; 75:685-705.

52. Dimri GP, et al. A biomarker that identifies senescent human cells in culture and in aging skin in vivo. Proc Natl Acad Sci U S A. 1995:92(20):9363-7.

53. Itahana K, Campisi J, Dimri GP. Methods to detect biomarkers of cellular senescence: the senescence-associated beta-galactosidase assay. Methods Mol Biol. 2007;371:21-31.

54. Franken NA, Rodermond HM, Stap J, Haveman J, van Bree C. Clonogenic assay of cells in vitro. Nat Protoc. 2006;1(5):2315-9.

55. Crowley LC, Waterhouse NJ. Measuring Survival of Hematopoietic Cancer Cells with the Colony-Forming Assay in Soft Agar. Cold Spring Harb Protoc. 2016;2016(8).

56. Volpe TA, et al. Regulation of heterochromatic silencing and histone H3 lysine-9 methylation by RNAi. Science. 2002;297(5588):1833-7.

57. Verdel $\mathrm{A}$, et al. RNAi-mediated targeting of heterochromatin by the RITS complex. Science. 2004;303(5658):672-6.

58. Nielsen $\mathrm{S}$, et al. Rb targets histone $\mathrm{H} 3$ methylation and HP1 to promoters. Nature. 2001;412(6846):561-5.

59. Schultz DC, Ayyanathan K, Negorev D, Maul GG, Rauscher FJ 3rd. SETDB1: a novel KAP-1-associated histone $\mathrm{H} 3$, lysine 9-specific methyltransferase that contributes to HP1-mediated silencing of euchromatic genes by KRAB zincfinger proteins. Genes Dev. 2002;16(8):919-32.

60. Jia S, Noma K, Grewal SI. RNAi-independent heterochromatin nucleation by the stress-activated ATF/CREB family proteins. Science. 2004;304(5679):1971-6.

61. Bulut-Karslioglu A, et al. A transcription factor-based mechanism for mouse heterochromatin formation. Nat Struct Mol Biol. 2012;19(10):1023-30.

62. Gonzalo S, et al. Role of the RB1 family in stabilizing histone methylation at constitutive heterochromatin. Nat Cell Biol. 2005;7(4):420-8.

63. Isaac CE, et al. The retinoblastoma protein regulates pericentric heterochromatin. Mol Cell Biol. 2006;26(9):3659-71.

64. Zhu Q, et al. BRCA1 tumour suppression occurs via heterochromatinmediated silencing. Nature. 2011;477(7363):179-84

65. Lomberk $\mathrm{G}$, et al. Sequence-specific recruitment of heterochromatin protein 1 via interaction with Kruppel-like factor 11, a human transcription factor involved in tumor suppression and metabolic diseases. J Biol Chem. 2012; 287(16):13026-39.

\section{Publisher's Note}

Springer Nature remains neutral with regard to jurisdictional claims in published maps and institutional affiliations. 